\title{
Efficacy and Tolerance Study of an Oligopeptide with Potential Anti-Aging Activity
}

\author{
Athanasia Varvaresou ${ }^{1}$, Spyridon Papageorgiou ${ }^{1}$, Evangelia Protopapa ${ }^{1}$, Alexandra Katsarou ${ }^{2}$ \\ ${ }^{1}$ Department of Aesthetics and Cosmetology, School of Health \& Caring Professions, Technological Educational Institution, Athens, \\ Greece; ${ }^{2}$ Department of Dermatology and Venereology, University of Athens, Athens, Greece, “Andreas Syggros" Venereal \& Skin \\ Diseases Hospital of Athens, Athens, Greece. \\ E-mail: avarvares@teiath.gr, varvaresou@pharm.uoa.gr
}

Received September $14^{\text {th }}, 2011$; revised November $1^{\text {st }}, 2011$; accepted November $14^{\text {th }}, 2011$.

\begin{abstract}
Background: Oligopeptide-20 acts as positive regulator of cell proliferation and increases collagen and hyaluronic acid levels in keratinocyte and fibroblast cultures. Methods: After randomization half the face of twenty volunteers was treated twice daily for two months with the oligopeptide-20 cream $(0.003 \% \mathrm{w} / \mathrm{w}, 300 \mathrm{ppm})$ and the other half with the vehicle. Skin bioengineering was used to estimate the effects on human skin. Results: The average number and width of wrinkles (Sew, $11.7 \%, p<0.05)$ and cyclic average roughness $\left(c R_{3}, 12.2 \%, p<0.005\right)$ were improved. Sesc parameter, indicative of stratum corneum dehydration was affected almost equally by the oligopeptide and the vehicle cream $(p<$ 0.1 ). Volunteers noted statistically significant improvement regarding fine wrinkles and skin brightness on the peptide treated side, whereas the skin smoothness was improved on both sides. No significant changes in pigmentation measured by Mexameter were recorded. No significant changes in transepidermal water loss values were demonstrated, indicating oligopeptide-20 does not affect the skin barrier. No serious adverse or side effects were observed. Additionally, the oligopeptide cream was not irritant according to $48 \mathrm{~h}$ semi-occluded patch test. Conclusion: Oligopeptide-20 could be a useful and safe ingredient of anti-aging cosmetics.
\end{abstract}

Keywords: Oligopeptide-20, Anti-Aging Cosmetics, Efficacy and Tolerance, Profilometry, Self-Evaluation, TEWL and Skin Barrier

\section{Introduction}

Peptides as cosmeceutical ingredients, evolved from research into wound healing and studies on growth stimulation of human skin fibroblasts $[1,2]$. In recent years there has been increased interest in the use of that signal peptides in the management of aging [3-5]. Palmitoyl pentapeptide-4 (Pal KTTKS) is a procollagen pentapeptide fragment [6] which stimulates collagen I and III and fibronectin production by fibroblasts in vitro and has been shown to decrease skin roughness and wrinkles and fine lines [7] after 4 month application. Copper tripeptide-1 is thought to improve skin firmness and texture, fine lines and hyperpigmentation [8]. Oligopeptide-20 is another synthetic peptide consisted of the following 12 amino acids: Arg-Arg-Leu-Glu-Met-Tyr-Cys-Ala-ProLeu-Lys-Pro [9]. It acts as positive regulator of cell proliferation and increases collagen and hyaluronic acid levels in keratinocyte and fibroblast cultures. In vitro decreases apoptosis rates and increases cell attachment, which is a significant part of wound healing and skin regeneration [10]. To the best of our knowledge, there is no study in the bibliography regarding the clinical efficacy of cosmetic products containing oligopeptide- 20 . As a part of our research program on anti-aging and cosmeceutical agents [11-15], we thought it would be of interest to examine by means of a randomized, vehicle controlled, double blind study the anti-aging properties of a cream containing $0.003 \% \mathrm{w} / \mathrm{w}(300 \mathrm{ppm})$ of the newly synthesized oligopeptide- 20 . We have used five different assessment methods to examine the effect of topical oligopeptide-20 treatment on the skin: Four noninvasive, bioengineering, objective techniques proposed by EEMCO Group (European Group in Efficacy Measurement of Cosmetics and Other Topical Products) and subjective self-evaluation. Skin surface morphology was examined by a direct non-invasive method with the instrument Skin VisioscanVC98 [16]. Skin microtopography was examined by optical profilometry (negative rep- 
lica method), which is a useful non-invasive method in trials estimating the effectiveness of anti-wrinkle preparations [17-22]. Pigmentation was examined by the melanin index measurement (Mexameter) [23,24]. The effect of the peptide on skin barrier was also investigated. Transepidermal water loss (TEWL) measurement can screen ingredients that have effect on barrier function and offer the possibility to monitor in vivo on human skin the effect of topical treatment in an objective and noninvasive way $[25,26]$. Additionally, assessment of skin tolerance of the oligopeptide- 20 cream was performed $[27,28]$.

\section{Materials and Methods}

\subsection{Treatment Creams}

Creams contained identical vehicle. The active cream contained oligopeptide- $200.003 \% \mathrm{w} / \mathrm{w}(300 \mathrm{ppm})$. Oligopeptide- 20 was donated by Cellco Chemicals Ltd (Athens, Greece), distributor in Greece of Caregen Co., Ltd (South Korea).

Ingredients: Aqua, C12-20 Acid PEG Ester, Glyceryl Stearate (and) PEG-100 Stearate, Cetyl Alcohol, Dimethicone, Octyl Palmitate, Xanthan Gum, Propylene Glycol, Propylparaben, Methylparaben, Imidazolidinyl Urea, BHT.

\subsection{Equipment}

Skin surface evaluation: Skin Visioscan VC98 (Courage and Khazaka, Cologne, Germany). Skin profilometry: Skin Visiometer SV600FW, Melanin content: Mexameter MX18 (MPA5, Courage and Khazaka, Cologne, Germany). Transepidermal water loss: Tewameter TM300 (Courage and Khazaka, Cologne, Germany).

\subsubsection{Efficacy Study}

\subsubsection{Volunteers}

Twenty females and two males (age $35-75$, mean age 55 years) were included in this study. They were selected according to following inclusion criteria:

Discontinuation of treatments by products containing retinoids, a-hydroxyacids and other anti-aging treatments at least six months before the beginning of the study.

\subsubsection{Signed Informed Consent}

Avoidance of medication such as steroid hormone replacement, birth control pills and vitamin supplements during the study period. At the baseline visit, the participants were instructed not to apply or take orally any other anti-wrinkle products/food supplements and not to apply at each visit, any product on the previous 12 hours.

\subsubsection{Study Procedure}

The procedure was done according to good clinical prac- tice (GCP) guidelines. Volunteers were visited at the baseline (D0) and D20, D40 and D60.

Skin surface evaluation, skin microtopography, melanin content, transepidermal water loss and were measured before (D0), during (D20, D40) and after the treatment (D60) and self-evaluation was performed by the test subject before (D0) and after 2-month treatment (D60). Side effects were also evaluated by the volunteers.

Two coded $50 \mathrm{~g}$ vessels, marked right and left, respectively for use twice daily on each half of the face were distributed at the first visit. At each control used vessels were collected in order to allow calculation of the amount of cream used. Tests were carried out in a halfface, double manner. The participants rested for a 20-min acclimatizing period before the measurements at a room temperature ranging between $19^{\circ} \mathrm{C}-21^{\circ} \mathrm{C}$ and $\mathrm{RH}$ between $40 \%$ and $60 \%$. The subject was seated in a comfortable armchair with closed eyes in order to relax. In this position measurements were done on the periocular skin surface.

\subsubsection{Skin Surface Evaluation}

The surface of living skin was analyzed by the direct non-invasive method using the instrument Skin Visioscan VC98 [16]. In Skin Visioscan technique the image of the skin is taken by a built in CCD-camera. The Surface Evaluation Living Skin (SELS) parameters Sew (wrinkles parameter), calculated from the average number and width of horizontal and vertical wrinkles and Sesc (scaliness parameter), representative of the dryness of stratum corneum, were evaluated [16]. The ideal moisturizing or anti-aging treatment should decrease Sew and Sesc.

\subsubsection{Skin Microtopography}

Skin Visiometer SV600FW (optical profilometry) is used for non-direct measurement of skin topography [17-22]. To obtain negative imprints of the examined skin area, fast hardened blue silicon and adhesive disks were used. The adhesive disk was layered onto the volunteer's skin to delimit the investigated area, avoiding skin stretching during the application and keeping track of the specimen orientation. A small amount of silicone was then spread into the internal circular area delimited by the adhesive disk and left in situ for 15 minutes until dry. The disc was next removed obtaining a careful duplicate of the skin surface on the silicone.

The silicon replica was put on a special frame and inserted in the special device where a light source sending out parallel light and a video sensor CCD on the other side. The replica reproduces the skin as a negative i.e. wrinkles are reproduced as mountains as the silicone is thicker in that place. Cyclic average roughness parameter 
$\left(\mathrm{cR}_{3}\right)$ was examined. $\mathrm{cR}_{3}$ is the average roughness calculated by circular arrangement of the lines in order to compensate the influence from the direction of the wrinkles [19]. The ideal moisturizing or anti-aging treatment should decrease $\mathrm{cR}_{3}$.

\subsubsection{Melanin Measurement}

Mexameter MX18 was used to estimate the activity of the oligopeptide on skin pigmentation $[23,24]$.

\subsubsection{Self-Assessment}

Subjects were asked to provide an assessment of improvement or not at the end of the treatment relative to baseline for fine lines, skin brightness and skin smoothness. These variables were scored on a scale of 1-4 indicating total (4), great (3), moderate (2), slight (1) and none $(0)$.

\subsubsection{Transepidermal Water Loss (TEWL)}

TEWL measurements $[25,26]$ were included to determine if the oligopeptide-20 affects skin barrier. Tewameter was used. In this instrument the water evaporation gradient, developed from the skin surface is measured by a probe that is placed perpendicularly on the skin site to be measured. The probe consists of an open cylinder containing two hygrosensors coupled with two thermistors placed at different distances from the skin surface. At both points, the relative humidity and temperature are measured and the corresponding vapour pressured is calculated. The difference between the vapour pressures at both points along the gradient is directly related to the rate of the evaporative water loss through the particular skin site.

\subsubsection{Statistical Analysis}

The improvement regarding Sew, Sesc and $\mathrm{cR}_{3}$ were calculated according to the equation [(ScoreDn - ScoreD0)/ Score D0] $\times 100$, where n: 20, 40 and 60 .

The Wilcoxon signed rank tests were applied to study variations between measurements obtained the first and last day of the study and paired $t$ tests between measurements of the active and vehicle side at D20, D40 and D60.

All the analyses were two-tailed. A p value $<0.05$ was considered statistically significant, whereas $p$ value $<0.1$ was considered suggestive and $\mathrm{p}<0.5$ side preferred.

The data was analyzed using the Statmost statistical package.

\subsubsection{Safety Evaluation}

In order to evaluate the tolerability and the potential irritant power of the cream containing oligopeptide-20, 13 subjects both females and males, with an age between 18 - 70 years have been selected for the test, following the under mentioned inclusion criteria: 1) good state of general health; 2) no dermatopathies; 3) no pharmacological treatment in progress; 4) promise not to change usual daily routine; 5) no atopy. The test involves single application, $48 \mathrm{~h}$ semi-occluded patch test. The equipment used for the semi-occluded patch is composed of a small cavity of $0.64 \mathrm{~cm}^{2}$ with a filter tissue at the bottom which is made to receive the product to test. All this is fixed to a hypoallergenic non woven adhesive tape. Treatment sites (back of the volunteers) are assessed before the first application of $0.02 \mathrm{ml}$ test material (baseline) and after treatment at 30 minutes after patch removal. Negative controls (empty patch) are used to facilitate evaluation. The quantification of the skin irritation is given through a numeric scale. The average irritant score of the product is calculated from the average of the quotations obtained for each volunteer, allowing ranking from "non irritant to very irritant". The reactions were evaluated according the following arbitrary scale [27,28].

Erythema

No erythema: 0, Light erythema (hardly visible): 1, Clearly visible erythema: 2, Moderate erythema: 3, Serious erythema (dark red with possible formation of light eschars): 4.

\section{Edema}

No edema: 0, Very light edema (hardly visible): 1, Light edema: 2, Moderate edema (about $1 \mathrm{~mm}$ raised skin): 3 , Strong edema (extended swelling even beyond the application area): 4.

The index of average irritation was classified according amended Draize system: Non irritating: 0.5, 0.5 - 2.0: slightly irritating, 2.0 - 5.0: moderately irritating, 5.0 8.0 (highly irritating).

\section{Results and Discussion}

Of those recruited in the ant-aging evaluation, 20 of 22 subjects completed the 2 month treatment period. The results of 20 subjects were analyzed. None abandoned the study for adverse events or serious side effects. The most common side effect reported was dryness (Table 1). Dryness remained during the 2 month treatment and has to be mainly attributed to the poor synthesis of both creams in emollient ingredients, since the above symptoms were recorded at almost equal ratios for both the active and vehicle cream. No irritant or allergic reactions were documented during the test period.

We used direct non-invasive Visioscan VC98 technique [16] in order to evaluate skin surface. Regarding our study, Sew parameter indicative of number and width of horizontal and vertical wrinkles showed a decrease of $11.7 \%(\mathrm{p}<0.05)$ in the case of active side. The decrease for the vehicle side was remarkably lower $(5.5 \%, \mathrm{p}<$ 0.05) (Figure 1). Sesc parameter, indicative of dryness of stratum corneum seemed to be affected almost equally 
Table 1. Protocol of side effects (\%) recorded on the active side and vehicle side by the subjects included in the trial. A test subject could report more than one side effect.

\begin{tabular}{lcccccc}
\hline & \multicolumn{2}{c}{ D20 } & \multicolumn{2}{c}{ D40 } & \multicolumn{2}{c}{ D60 } \\
\cline { 2 - 6 } & Active & Vehicle & Active & Vehicle & Active & Vehicle \\
\hline A. No side-effect & 0 & 0 & 0 & 0 & 0 & 0 \\
B. Feeling of warmth in the skin & 5 & 5 & 0 & 0 & 0 & 0 \\
C. Stinging & 0 & 0 & 0 & 0 & 0 & 0 \\
D. Redness & 5 & 0 & 0 & 0 & 0 & 0 \\
E. Desquamation & 0 & 0 & 0 & 0 & 0 & 0 \\
F. Dryness & 50 & 50 & 20 & 50 & 20 & 20 \\
G. Itching & 0 & 0 & 0 & 0 & 0 & 0 \\
H. Ocular intolerance & 0 & 0 & 0 & 0 & 0 & 0 \\
\hline
\end{tabular}

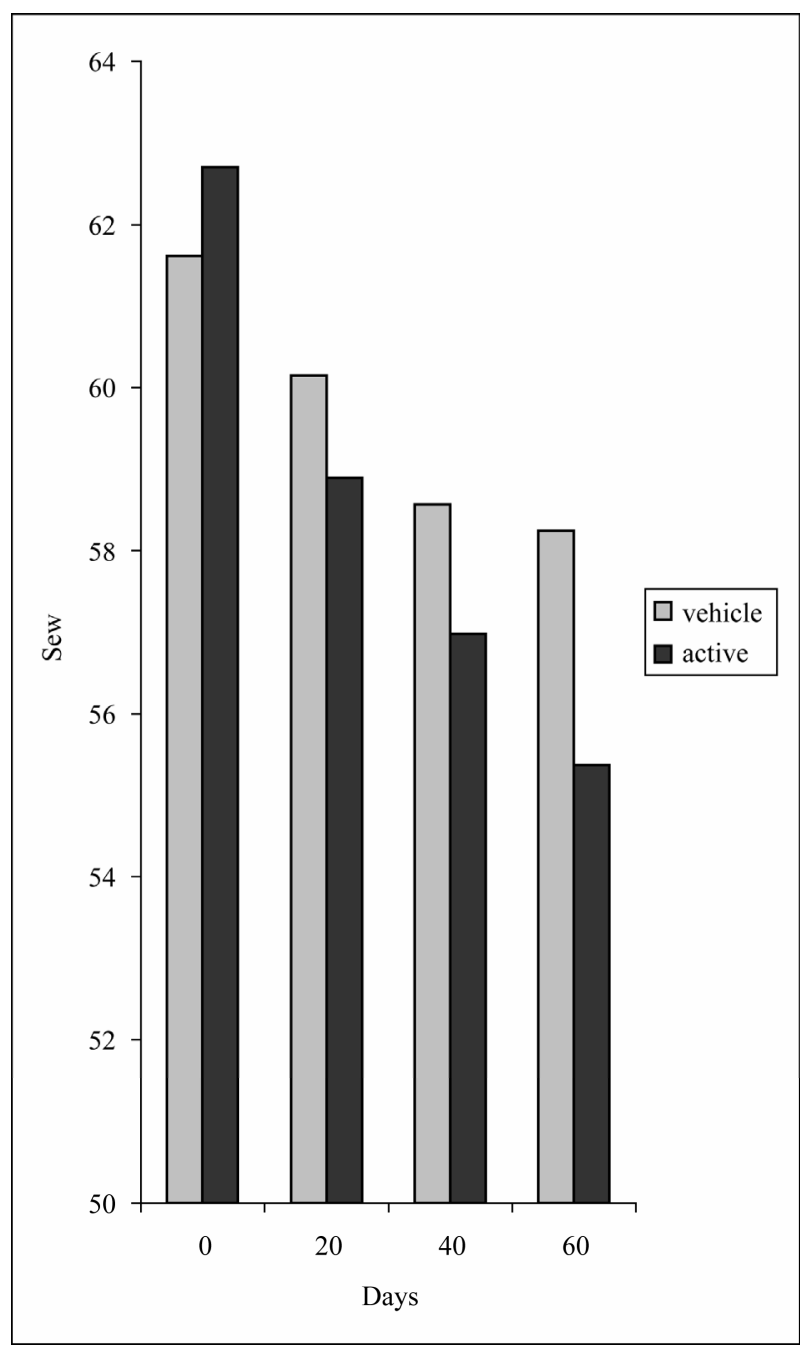

Figure 1. Comparison between Sew values measured at each time point (D0, D20, D40 and D60). p $<0.05$ Wilcoxon test for vehicle; $\mathrm{p}<\mathbf{0 . 0 5}$ Wilcoxon test for active. by the oligopeptide and the vehicle cream in a rather statistically suggestive manner $(\mathrm{p}<0.1)$ (Figure 2).

We used optical profilometry (negative skin replicas) in order to evaluate skin microtopography, as well [17$22]$. To date most of the non-invasive methods of quantification of cutaneous relief have consisted of the replicas. The relief profile is measured by optical techique. The metal industry has for several years defined and standardized the parameters used for characterization of the surface area of the metals. These parameters are the ones used for profilometric analysis of cutaneous microrelief.

The major parameters cited in the literature for skin surface are R1, R2 and R3 [19]. The measuring of these parameters is of importance for the discussion of the actual efficacy of the products on wrinkles and skin roughness, although clinical diversity and skin microrelief and wrinkles can hardly be reduced to the measurement of two or three parameters. As age increased, an increase of roughness parameters is observed [19].

In our study, a gradual improvement of cyclic roughness $\left(\mathrm{cR}_{3}\right)$ was observed during treatment for both sides. The $\mathrm{cR}_{3}$ showed an overall decrease of $12.2 \%$ in the case of active side, whereas the decrease was almost the half (6.4\%) for the vehicle side (Figure 3).

Regarding pigmentation, no identified influence on melanin content was recorded either in peptide- or vehicle-treated half face.

In the self-assessment $85 \%$ of the subjects ( 17 of $20, p$ $<0.005)$ claimed that the active side showed different degrees of improvement in fine wrinkles with a median of 1.35 (slight to moderate) while $65 \%$ (13 of 20, p < 0.005 ) claimed the vehicle treated side had improved with a median of 0.75 (rather slight). $80 \%$ of the subjects (16 of 20, p $<0.005$ ) reported slight improvement of skin brightness in active side, while $55 \%$ (11 of $20, \mathrm{p}<0.005)$ 


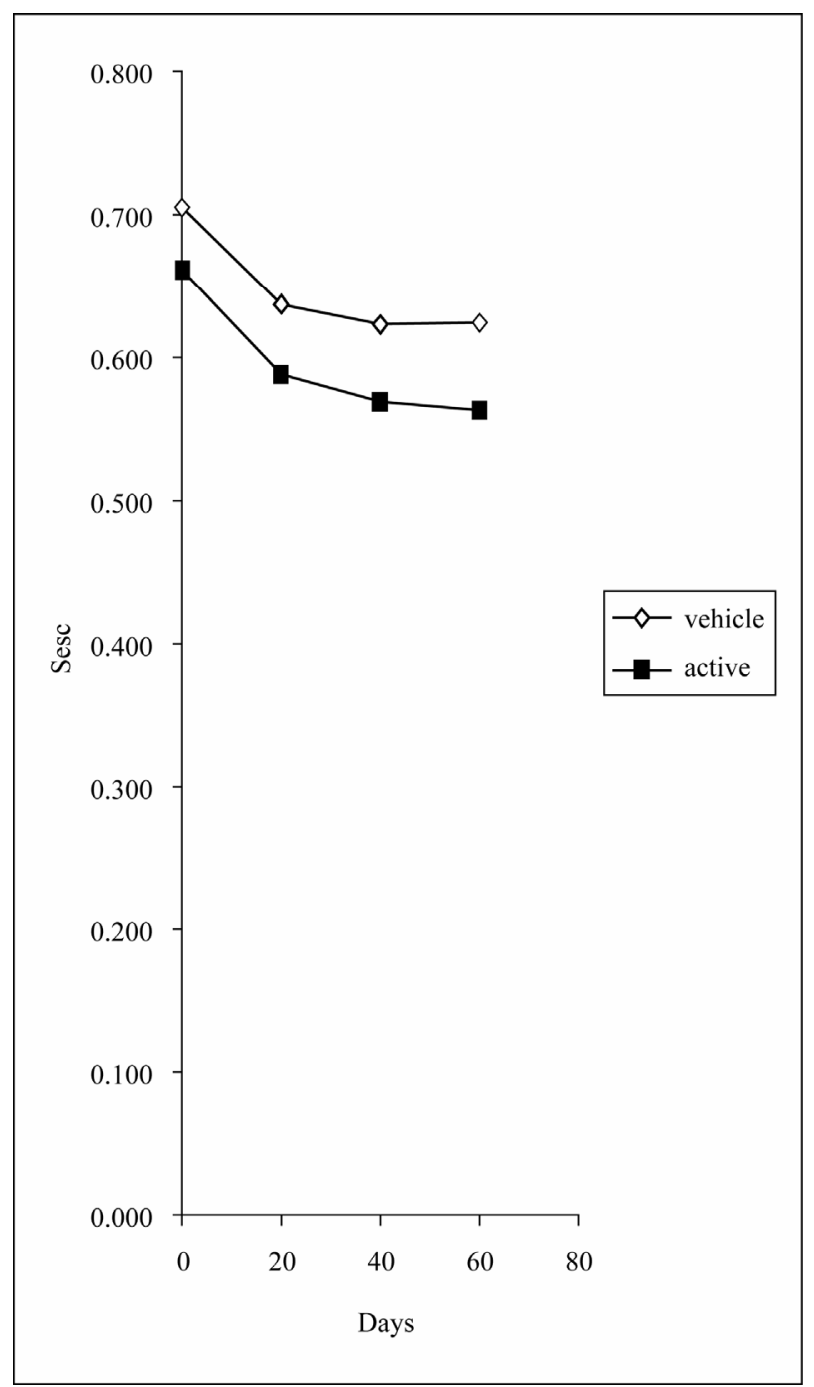

Figure 2. Sesc measurements for the active and vehicle sides at each time point (D0, D20, D40 and D60). p $<0.1$ Wilcoxon test for vehicle; $p<0.1$ Wilcoxon test for active.

claimed none to slight improvement (median 0.55) in the vehicle side (Table 2). The results of self-assessment were in accordance with the data obtained by the bioengineering measurement. The recorded improvement of fine wrinkles by the $85 \%$ of the volunteers on the peptide-treated half correlates well with the decrease in wrinkles (Sew, D60, $\mathrm{p}<0.05$ ) and decrease in roughness $\left(\mathrm{cR}_{3}, \mathrm{D} 60, \mathrm{p}<0.005\right)$. Only the slight improvement in skin brightness recorded by $80 \%$ of the volunteers on the active side is not confirmed by the Mexameter measurements, since, the treatment did not reduce the extent and number of hyperpigmented spots on the face nor changed skin color as was measured by Mexameter. These subject-perceived benefits may be due to a "halo-effect" - subjects received a desired skin improvement on the peptide-treated side and translated that to a total improve

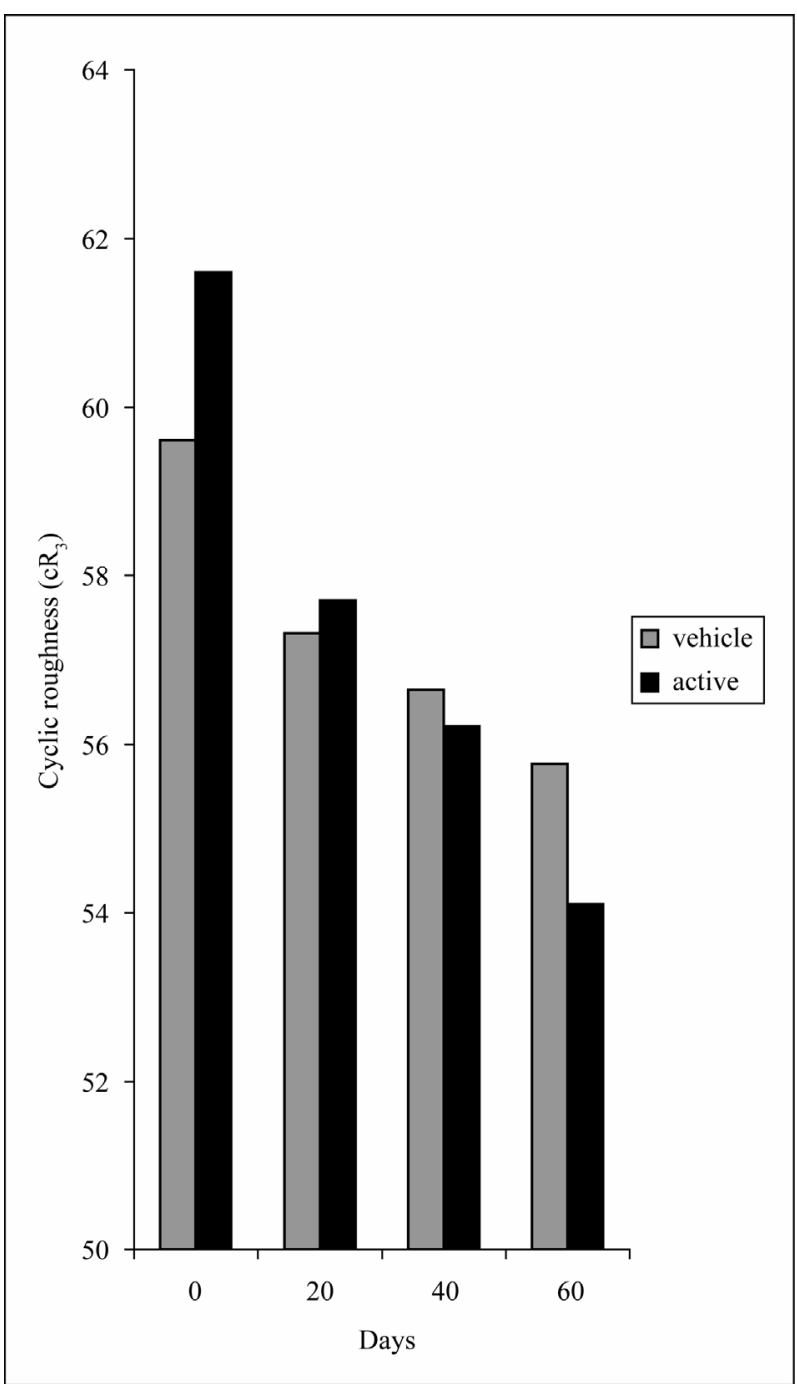

Figure 3. Comparison between $\mathrm{cR}_{3}$ values measured at each time point (D0, D20, D40 and D60). p $<0.005$ Wilcoxon test vehicle and active.

ment across benefit areas. Therefore, the combination of self-assessment with the bioengineering methods seems necessary.

Disturbances in biophysical properties of the skin during irritant reaction induced by exposure to cosmetic ingredients may be assessed by several non-invasive methods. The most popular one involves determinations of transepidermal water loss (TEWL), stratum corneum hydration, skin $\mathrm{pH}$ and evaluation of erythema and edema and skin blood flow [29]. No significant changes in transepidermal water loss values were demonstrated by either active or vehicle cream indicating oligopeptide- 20 does not affect the skin barrier function.

The possible irritating power of the tested peptide was evaluated according single application, $48 \mathrm{~h}$ semioccluded patch test. Neither erythema or edema after the 
Table 2. Subjective evaluation at D60.

\begin{tabular}{|c|c|c|c|c|c|c|}
\hline \multirow{2}{*}{ Volunteer } & \multicolumn{2}{|c|}{ Fine Lines } & \multicolumn{2}{|c|}{ Skin Brightness } & \multicolumn{2}{|c|}{ Skin Smoothness } \\
\hline & Active side & Vehicle side & Active side & Vehicle side & Active side & Vehicle side \\
\hline 1 & 2 & 0 & 1 & 1 & 2 & 2 \\
\hline 2 & 1 & 0 & 1 & 1 & 2 & 2 \\
\hline 3 & 2 & 0 & 0 & 1 & 2 & 2 \\
\hline 4 & 1 & 1 & 1 & 1 & 1 & 2 \\
\hline 5 & 2 & 1 & 2 & 0 & 2 & 1 \\
\hline 6 & 2 & 0 & 0 & 0 & 1 & 1 \\
\hline 7 & 0 & 0 & 1 & 0 & 2 & 1 \\
\hline 8 & 1 & 0 & 1 & 1 & 2 & 2 \\
\hline 9 & 0 & 1 & 0 & 1 & 1 & 1 \\
\hline 10 & 2 & 1 & 1 & 0 & 2 & 1 \\
\hline 11 & 1 & 1 & 0 & 0 & 1 & 1 \\
\hline 12 & 2 & 2 & 1 & 1 & 2 & 2 \\
\hline 13 & 1 & 1 & 2 & 1 & 1 & 2 \\
\hline 14 & 0 & 0 & 1 & 0 & 2 & 2 \\
\hline 15 & 1 & 1 & 1 & 1 & 2 & 1 \\
\hline 16 & 2 & 2 & 2 & 1 & 2 & 1 \\
\hline 17 & 1 & 1 & 1 & 0 & 2 & 1 \\
\hline 18 & 2 & 1 & 1 & 1 & 1 & 1 \\
\hline 19 & 2 & 1 & 1 & 0 & 2 & 1 \\
\hline 20 & 2 & 1 & 2 & 0 & 2 & 1 \\
\hline Total score & 27 & 15 & 20 & 11 & 34 & 28 \\
\hline Median score* & 1.35 & 0.75 & 1 & 0.55 & 1.7 & 1.4 \\
\hline Percentage** & 85 & 65 & 80 & 55 & 100 & 100 \\
\hline $\mathrm{p}^{* * *}$ & \multicolumn{2}{|c|}{$<0.005$} & \multicolumn{2}{|c|}{$<0.005$} & \multicolumn{2}{|c|}{$<0.5$} \\
\hline$\frac{\text { total score }}{\text { Number of volu }}$ & $* * N$ & \multicolumn{3}{|c|}{$* * \frac{\text { Number of volunteers reported improvement }}{2} \times 10$} & & tailed $t$ test be- \\
\hline
\end{tabular}

removal was recorded (Table 3).

Further studies to elucidate possible mechanism of action and optimal dosage treatment periods are warranted. Although, the penetration and localization of the liposome form of oligopeptide- 20 has been captured by fluorescence microscope, the labeled peptide being observed in epidermis and dermis [10], cutaneous penetration of pure oligopeptide will be in the future examined. At present we do not know which the ideal treatment period is. We think that a 4-month follow-up study would be useful in order to ensure the maximum improvement The $0.003 \% \mathrm{w} / \mathrm{w}$ concentration of oligopeptide- 20 in the cream used in the parent study is according to empirical data and is lower than maximum recommended $(0.005 \%$ $\mathrm{w} / \mathrm{w}, 500 \mathrm{ppm})$.

In conclusion, the application of the tested cream with $0.003 \% \mathrm{w} / \mathrm{w}$ oligopeptide-20 improves several clinical characteristics relating to skin aging, without damage to skin barrier or serious side effects. Furthermore, it is not irritating. The addition of moisturizing and emollient 
Table 3. Values and classification of average irritation indexes.

\begin{tabular}{cccc}
\hline Volunteer & Erythema & Edema & $\begin{array}{c}\text { Total readings } \\
48 \mathrm{hr}\end{array}$ \\
\hline 1 & 0 & 0 & 0 \\
2 & 0 & 0 & 0 \\
3 & 0 & 0 & 0 \\
4 & 0 & 0 & 0 \\
5 & 0 & 0 & 0 \\
6 & 0 & 0 & 0 \\
7 & 0 & 0 & 0 \\
8 & 0 & 0 & 0 \\
9 & 0 & 0 & 0 \\
10 & 0 & 0 & 0 \\
11 & 0 & 0 & 0 \\
12 & 0 & 0 & 0 \\
13 & 0 & 0 & 0 \\
Total irritation & & & 0 \\
Result & & & 0 \\
\hline
\end{tabular}

agents could enhance the anti-wrinkle activity of the oligopeptide and lead to the development of effective, innovative and safe formulations.

\section{Acknowledgements}

A part of the work was supported by Technological Educational Institution of Athens through "THALIS" action. We also would like to thank Caregen Co., (South Korea) manufacturer of oligopeptide-20 and the distributor in Greece, Cellco Chemicals Ltd. (Athens, Greece) for providing us with the oligopeptide- 20 .

\section{REFERENCES}

[1] R. A. F. Clark, "Overview and General Consideration," In: R. A. F. Clark, Ed., The Molecular and Cellular Biology of Wound Repair, Plenum Press, New York, 1995, pp. 3-50. doi:10.1007/978-1-4615-1795-5

[2] A. Siméon, Y. Wegorawski and Y. Bontemps, "FrancçoisXavier Maquart: Expression of Glycosaminoglycans and small Proteoglycans in Wounds: Modulation by the Tripeptide-Copper Complex Glycyl-L-Histidyl-L-Lysine-Cu ${ }^{2+}$," Journal of Investigative Dermatology, Vol. 115, No. 6, 2000, pp. 962-968. doi:10.1046/j.1523-1747.2000.00166.x

[3] M. P. Lupo, “Cosmeceutical Peptides,” Dermatologic Sur- gery, Vol. 31, Supplement 1, 2005, pp. 832-836. doi:10.1111/j.1524-4725.2005.31728

[4] F. Gorouhi and H. I. Maibach, "Role of Topical Peptides to Preventing or Treating Aged Skin," International Journal of Cosmetic Science, Vol. 31, No. 5, 2009, pp. 327345. doi:10.1111/j.1468-2494.2009.00490.x

[5] Z. D. Draelos, "Cosmeceuticals Realm," Clinics in Dermatology, Vol. 26, No. 6, 2008, pp. 627-632. doi:10.1016/j.clindermatol.2007.09.005

[6] K. Katayama, J. Armendariz-Borunda, R. Raghow, A. H. Kang and J. M. Seyer, "A Pentapeptide from Type I Procollagen Promotes Extracellular Matrix Production," The Journal of Biological Chemistry, Vol. 268, No. 14, 1993, pp. 9941-9944.

[7] L. R. Robinson, N. C. Fitzgerald, D. G. Doughty, N. C. Dawes, C. A. Berge and D. L. Bisset, "Topical Palmitoyl Pentapeptide Provides Improvement in Photoaged Human Facial Skin," International Journal of Cosmetic Science, Vol. 27, No. 3, 2005, pp. 155-160. doi:10.1111/j.1467-2494.2005.00261.x

[8] M. B. Finkey, Y. Appa and S. Bhandarkar, "Copper Peptide and Skin," In: P. Elsner and H. Maibach, Eds., Cosmeceuticals and Active Cosmetics, Taylor \& Francis, New York, 2005, pp. 549-564.

[9] US Patent No. 0169491, 2009.

[10] Caregen Co. Ltd. Formulary, 2006.

[11] A. Varvaresou, E. Tsaoula and E. Tsirivas, "The Influence of the Addition of Melatonin to a Magnesium Ascorbyl Phosphate-Containing Formulation on the Mechanical Properties of Human Skin," Review of Clinical Pharmacology and Pharmacokinetics International Edition, Vol. 18, No. 3, 2004, pp. 177-181.

[12] A. Varvaresou, E. Tsirivas, E. Tsaoula and E. Protopapa, "The Influence of Magnesium Ascorbyl Phosphate on Moisturization of Stratum Corneum," Review of Clinical Pharmacology and Pharmacokinetics International Edition, Vol. 19, No. 3, 2005, pp. 139-142.

[13] S. Papageorgiou, A. Varvaresou and E. Tsirivas, "The Development of Self-Preserving Cosmetics," Review of Clinical Pharmacology and Pharmacokinetics International Edition, Vol. 22, No. 3, 2008, pp. 455-460.

[14] A. Varvaresou, S. Papageorgiou, E. Tsirivas, E. Protopapa, H. Kintziou, V. Kefala and C. Demetzos, "SelfPreserving Cosmetics," International Journal of Cosmetic Science, Vol. 31, No. 3, 2009, pp. 163-175. doi:10.1111/j.1468-2494.2009.00492.x

[15] S. Papageorgiou, A. Varvaresou and C. Demetzos, "New Alternatives to Cosmetics Preservation," Journal of Cosmetic Science, Vol. 61, No. 2, 2010, pp. 107-123.

[16] M. R. Ferreira Pena, P. C. Costa and F. M. Bahia, "Efficacy of Anti-Wrinkle Products in Skin Surface Appearance: A Comparative Study Using Non-Invasive Methods," Skin Research and Technology, Vol. 16, No. 4, 2010, pp. 444-449. doi:10.1111/j.1600-0846.2010.00458.x

[17] J. Hatzis, "The Wrinkle and Its Measurement-A Skin Surface Profilometric Method," Micron, Vol. 35, No. 3, 
2004, pp. 201-219. doi:10.1016/i.micron.2003.11.007

[18] J. L. Lévêque, "EEMCO Guidance for the Assessment of Skin Topography. The European Expert Group on Efficacy Measurement of Cosmetics and Other Topical Products," Journal of the European Academy of Dermatology, Vol. 12, No. 2, 1999, pp. 103-114.

[19] H. K. Lee, Y. K. Seo, J. H. Baek and J. S. Koh, "Comparison between Ultrasonography (Dermascan C Version 3 ) and Transparency Profilometry (Skin Visiometer SV600)," Skin Research and Technology, Vol. 14, No. 1, 2008, pp. 8-12.

[20] M. Udompataikul, P. Sripoj and P. Palungwachira, “An Oral Nutraceutical Containing Antioxidants, Minerals and Glycosaminoglycans in Skin Roughness and Fine Wrinkles," International Journal of Cosmetic Science, Vol. 31, No. 6, 2009, pp. 427-435. doi:10.1111/j.1468-2494.2009.00513.x

[21] G. L. Grove, M. J. Grove and J. J. Leyden, "Optical Profilometry: An Objective Method for Quantification of Facial Wrinkles," Journal of the American Academy of Dermatology, Vol. 21, No. 3, 1989, pp. 631-637. doi:10.1016/S0190-9622(89)70230-9

[22] M. Loden, I. Buraczewska and K. Halvarsson, "Facial Anti-Wrinkle Cream: Influence of Product Presentation on Effectiveness: A Randomized Controlled Study," Skin Research and Technology, Vol. 13, No. 2, 2007, pp. 189194. doi:10.1111/j.1600-0846.2007.00220.x

[23] P.-C. Chiu, C.-C. Chan, H.-M. Lin and H.-C. Chiu, "The Clinical Anti-Aging Effects of Topical Kinetin and Niacinamide in Asians: A Randomized, Double-Blind, Placebo-Controlled, Split-Face Comparative Study," Journal of Cosmetic Dermatology, Vol. 6, No. 4, 2007, pp. 243-249. doi:10.1111/j.1473-2165.2007.00342.x
[24] S. Y. Huh, J. W Shin, J. I. Na, C. H. Huh, S. W. Youn and K. C. Park, "Efficacy and Safety of Liposome-Encapsulated 4-N-Butylresorcinol $0.1 \%$ Cream for the Treatment of Melasma: A Randomized Controlled Split-Face Trial," The Journal of Dermatology, Vol. 37, No. 4, 2010, pp. 311-315. doi:10.1111/j.1346-8138.2010.00787.x

[25] C. Rosado, P. Pinto and L. M. Rodrigues, "Comparative Assessment of the Performance of Two Generations of Tewameter: TM210 and TM300," International Journal of Cosmetic Science, Vol. 27, No. 4, 2005, pp. 237-241. doi:10.1111/j.1467-2494.2005.00270.x

[26] V. Rogiers and the EEMCO Group, "EEMCO Guidance for the Assessment of Transepidermal Water Loss in Cosmetic Sciences," Skin Pharmacology and Applied Skin Physiology, Vol. 14, No. 2, 2001, pp. 117-128. doi:10.1159/000056341

[27] A. Katsarou, M. Kalogeromitros, V. Armenaka, E. Koufou, E. Davou and E. Koumantaki, "Trends in the Results of Patch Testing to Standard Allergens," Contact Dermatitis, Vol. 37, No. 5, 1997, pp. 245-246. doi:10.1111/j.1600-0536.1997.tb02452.x

[28] M. Milingou, A. Tagka, N. Armenaka, K. Kimpouri, D. Koimintzis and A. Katsarou, "Patch Tests in Children: A Review of 13 Years of Experience in Comparison with Previous Data," Pediatric Dermatology, Vol. 27, No. 3, 2010, pp. 255-259. doi:10.1111/j.1525-1470.2010.01128.x

[29] J. W. Fluhr, R. Darlenski, I. Angelova-Fisher, N. Tsankov and D. Basketter, "Skin Irritation and Sensitization: Mechanisms and New Approaches for Risk Assessment," Skin Pharmacology and Physiology, Vol. 21, No. 3, 2008, pp. 124-135. doi:10.1159/000131077 